\title{
Universiteit
}

Leiden

The Netherlands

\section{Triggering a Phase Transition by a Spatially Localized Laser Pulse: Role of Strain}

Bedoui, S.; Lopes, M.; Nicolazzi, W.; Bonnet, S.A.; Zheng, S.; Molnar, G.; Bousseksou, A.

\section{Citation}

Bedoui, S., Lopes, M., Nicolazzi, W., Bonnet, S. A., Zheng, S., Molnar, G., \& Bousseksou, A. (2012). Triggering a Phase Transition by a Spatially Localized Laser Pulse: Role of Strain. Physical Review Letters, 109(13), 135702. doi:10.1103/PhysRevLett.109.135702

Version: $\quad$ Not Applicable (or Unknown)

License: $\quad$ Leiden University Non-exclusive license

Downloaded from: https://hdl.handle.net/1887/61365

Note: To cite this publication please use the final published version (if applicable). 


\title{
Triggering a Phase Transition by a Spatially Localized Laser Pulse: Role of Strain
}

\author{
Salma Bedoui, ${ }^{1}$ Manuel Lopes, ${ }^{1}$ William Nicolazzi, ${ }^{1}$ Sylvestre Bonnet, ${ }^{2}$ Sipeng Zheng, ${ }^{2}$ \\ Gábor Molnár, ${ }^{1, *}$ and Azzedine Bousseksou ${ }^{1, \dagger}$ \\ ${ }^{1}$ Laboratoire de Chimie de Coordination, CNRS UPR-8241 and Université de Toulouse, UPS, INPT, F-31077, Toulouse, France \\ ${ }^{2}$ Leiden Institute of Chemistry, Leiden University, 2300 RA Leiden, Netherlands
}

(Received 9 July 2012; published 28 September 2012)

\begin{abstract}
We report here the optical microscopic imaging of a first-order phase transition induced by a nanosecond laser pulse $(532 \mathrm{~nm})$ in a single crystal of the molecular spin-crossover complex [ $\mathrm{Fe}($ bapbpy $\left.)(\mathrm{NCS})_{2}\right]$. The transition starts with the formation of a high spin domain in the region irradiated by the focused laser beam, followed by the subsequent growth or contraction of the initial domain. Remarkably, in otherwise identical experimental conditions one can observe either the irreversible transition of the whole crystal or merely the formation of a transient domain - depending on which region of the crystal is excited. This observation as well as the rather slow dynamics suggest that the main control parameter is the inhomogeneous accommodation strain, which destabilizes the photoinduced domain.
\end{abstract}

PACS numbers: 64.70.K-, 07.60.Pb, 64.60.My, 75.30.Wx

Photoinduced phase transition (PIPT) phenomena have received much attention in various types of solids such as organic charge transfer compounds, metal oxides, inorganic complexes, and even in metals [1-4]. In these materials, the strong electron-lattice or spin-lattice interactions have been shown to play a key role in driving the photoinduced transformation. Experimental evidence indicating that relatively weak photoexcitation can trigger a real macroscopic phase transition has been inferred in these systems from the huge photoconversion efficiencies as well as from the occurrence of characteristic nonlinear phenomena such as a threshold behavior. Here, we report on a conceptually different investigation where the phase transition was triggered by a spatially localized laser pulse within a comparatively large single crystal. Compared to previous studies on PIPT phenomena, our work brings in two new experimental features: (1) only a small part of the crystal is photoexcited by the focused laser beam, and (2) the phase change is not averaged for the whole crystal by a point or spectroscopic array detector but followed instead by an imaging charge-coupled device.

For our experiments, we have chosen the molecular spin-crossover compound $\left[\mathrm{Fe}^{\mathrm{II}}(\right.$ bapbpy $\left.)(\mathrm{NCS})_{2}\right] \quad$ (1) (where bapbpy $=N$ - $\{6$-[6-(pyridin-2-ylamino)pyridin-2yl]pyridin-2-yl $\}$ pyridin-2-amine). Spin-crossover (SCO) materials of transition metal complexes are benchmark examples for PIPT. They exhibit bistability between the so-called low spin (LS) and high spin (HS) electronic configurations, which display strikingly different physical properties, including mass density, magnetic susceptibility, optical density, etc. [5]. Switching between the two molecular spin states can be induced by changing the sample temperature, applying external pressure or an intense magnetic field, and also by light irradiation. Light-induced excited spin state trapping in the solid state was first demonstrated by Decurtins et al. [6]. This so-called
"LIESST effect" is basically a molecular phenomenon and can be observed only at cryogenic temperatures. On the other hand, several studies aimed also to achieve PIPT in spin-crossover solids at the macroscopic scale using short laser pulses [7-16]. It was shown that a single laser pulse (ns or fs) can drive the system from one spin state to the other, but the microscopic details of this phenomenon remain largely unexplored-in particular for the most interesting cooperative systems.

Compound $\mathbf{1}$ is a mononuclear iron(II) complex displaying a two-step thermal and pressure-induced spin transition [17-21]. Both steps involved in the transition are first order and show wide hysteresis loops of 6 and $25 \mathrm{~K}$ at atmospheric pressure. The ordered intermediate phase (IP) is characterized by a repetition of a (LS-LS-HS) motif-leading to a tripling of the volume of the monoclinic $(C 2 / c)$ unit cell. This phase is stable for a wide temperature range of $\sim 50 \mathrm{~K}$. At lower temperature, the IP is transformed to the triclinic LS phase. Since this second transition involves a considerable deformation of the unit cell and also twining, all thermal cycles in the present study were limited to the temperature range between 273 and $223 \mathrm{~K}$, i.e., around the high-temperature hysteresis loop (Fig. 1). Upon cooling, the color of the crystals changes from light red to dark red due to the higher absorbance of the LS molecules in the visible spectral range. This change of optical density offers a straightforward means to follow the spin state changes in a quantitative manner by optical microscopy [20-22].

Single crystals of $\mathbf{1}$ were synthesized using the method described in Ref. [17]. The variable-temperature optical microscopy experiments presented in this Letter were carried out using the same crystal $\left(c a .30 \times 20 \times 20 \mu \mathrm{m}^{3}\right.$ size) and the same optical microscopy setup as described in Ref. [21]. Figure 2(a) displays selected transmitted light microscopy images of the crystal recorded in the heating mode during the IP $\rightarrow$ HS transition. At $237 \mathrm{~K}$, the whole 


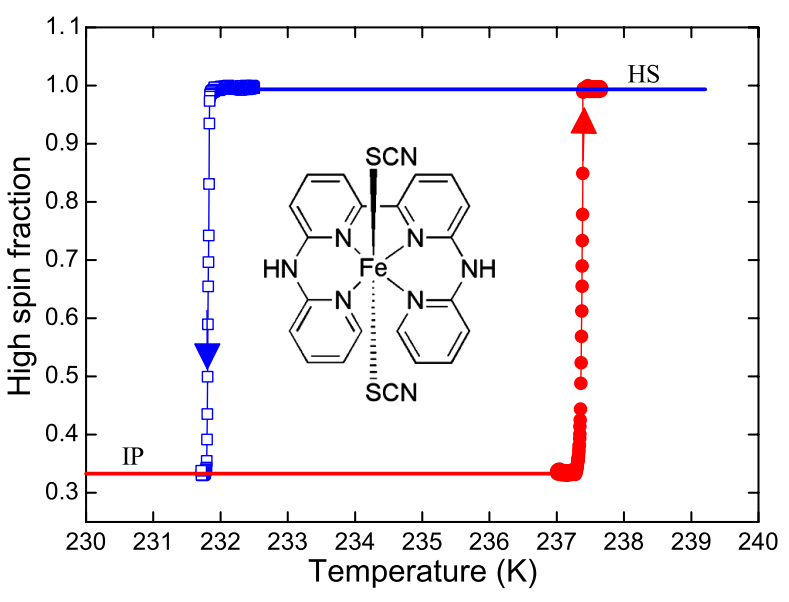

FIG. 1 (color online). Thermal hysteresis loop associated with the spin transition between the HS and the intermediate (LS-LSHS) phases in a single crystal of $\mathbf{1}$. Open and closed symbols indicate the cooling and heating modes $(d T / d t=0.5 \mathrm{~K} / \mathrm{min})$, respectively. Lines are inserted to guide the eye. The molecular structure of the complex is shown in the inset.

crystal is in the IP. Around $237.3 \mathrm{~K}$, the nucleation of the HS phase is observed simultaneously at two different places: in the bottom-left corner and at the top edge of the crystal. However, the new phase grows chiefly from the bottom nucleation site, first along the short axis and then along the long axis of the crystal with a mean velocity of ca. 3-4 $\mu \mathrm{m} / \mathrm{s}$ [Fig. 2(c)]. These observations have been reproduced several times; i.e., the physical properties and

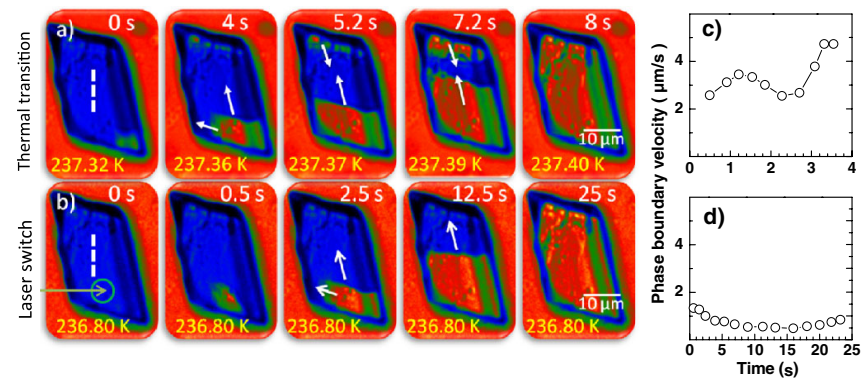

FIG. 2 (color online). Spatiotemporal development of the thermal and laser-induced spin transitions. (a) Bright-field, transmitted light optical microscopy snapshots of a crystal of $\mathbf{1}$ during the thermal transition in the heating mode $(d T / d t=$ $0.5 \mathrm{~K} / \mathrm{min}$ ). (b) Isothermal images of the same crystal at $236.8 \mathrm{~K}$ (just below the thermal transition) before and after a single laser shot ( $4 \mathrm{~ns}, 532 \mathrm{~nm}, 0.1 \mu \mathrm{J}, 3 \mu \mathrm{m}$ spot size). The circle indicates the area irradiated by the laser at $t=0$, and the arrows depict the phase propagation direction. Dark gray (blue) and light gray (red) (artificial) colors of the crystal refer to the IP and the HS phase, respectively. (The movies are available in the Supplemental Material [23].) The right panel depicts the instantaneous phase boundary velocities (c) during the thermal spin transition and (d) during the laser-induced transition. The CCD pixels for which the analysis was carried out are shown in the images by dashed lines. integrity of these crystals remain virtually unaffected during thermal cycling. In agreement with our earlier findings [20], the nucleation and subsequent growth during the high-temperature transition in $\mathbf{1}$ occur thus in a predetermined way. It starts (in most cases) at the edge of the crystal, presumably at a preferential defect site, where the energy barrier for the nucleation is lower. Such defects in different crystals are formed during the crystal growth in an arbitrary way-explaining why the transition curves differ somewhat from one crystal to other. The subsequent growth of the new phase is anisotropic and often discontinuous. The former observation is obviously related to the anisotropy of the crystal structure and crystal properties, while the latter is a consequence of sample inhomogeneityincluding defects and stresses in the crystal [20].

In the next experiment, the sample was heated only to $236.8 \mathrm{~K}$, i.e., before the nucleation of the HS phase could occur, and it was stabilized at this temperature during several minutes. Then, the crystal was exposed to a single focused nanosecond laser shot $(532 \mathrm{~nm}, 4 \mathrm{~ns}, 0.1 \mu \mathrm{J}, 3 \mu \mathrm{m}$ spot size), while it was imaged as a function of time at constant temperature. As shown in Fig. 2(b), the laser pulse first transforms locally a small volume of the crystal into the HS state. This step occurs within the time resolution of our camera $(<10 \mathrm{~ms})$ and it is followed by the conversion of the bottom-left corner of the crystal. The subsequent growth of the HS phase resembles closely the thermal transitionexcept that no nucleation occurs in this case at the top edge. At the end, ca. $25 \mathrm{sec}$ after the laser shot, the whole crystal is transformed to the HS state. This phenomenon was perfectly reproducible and it was observed on several other crystals as well (see the Supplemental Material for further examples [23]). As a general trend, we found that the total conversion of the crystal can be triggered more efficiently close to the edges and it has the highest probability when the laser pulse is shined in the vicinity of the "native nucleation site." This finding is consistent with the fact that these sites correspond to the lowest nucleation barrier in the crystal. In many cases, we observed also an apparent incubation period of several seconds between the formation of the laser-induced HS domain and the subsequent growth process (see the Supplemental Material [23]).

One shall note here that some laser-induced heating always occurs in the sample. This can explain-at least partly - the formation of the initial HS domain, which takes place below the time resolution of our experiment $(<\sim 10 \mathrm{msec})$. On the other hand, the further growth of this HS domain lasts several seconds. The duration of this process is thus several orders of magnitude longer than the time needed for the heat dissipation, which is completed within a few milliseconds [11,15]. This means thatcontrary to the nucleation - the propagation of the HS phase through the crystal cannot be related to laser heating but is instead a genuine cooperative phenomenon.

We have investigated in more detail the propagation kinetics of the HS phase using a similar analysis as that 
reported in Ref. [22], i.e., by examining the local kinetic curves along the phase boundary propagation direction. A remarkable feature of the phase propagation is the virtually constant and very slow velocity of the phase boundary [Figs. 2(c) and 2(d)]. The mean velocity is lower for the laser-induced transition when compared to the thermal transition $(0.8$ vs $3.4 \mu \mathrm{m} / \mathrm{s})$, but the two experiments differ in many details, the main difference being the sample temperature. We shall underline that this rather slow switching process in cooperative SCO materials follows several faster steps in the multiscale spatiotemporal dynamics, including ultrafast local photo switching (ps), elastic switching (ns), and thermal switching $(\mu \mathrm{s})[11,12]$. These faster switching processes are ubiquitous for each bulk SCO system, including noncooperative ones. On the other hand, we show here that the phase separation process gives rise to a further step in the switching dynamics, related to the phase boundary motion(s). This process is driven primarily by the elastic stress [22], but controlled in a complex manner by the movement of dislocations as well as by the presence of latent heat [24].

Interestingly, when the same laser shot was applied at the same temperature $(236.8 \mathrm{~K})$ but in the middle of the same crystal (Fig. 3), we observed reproducibly the formation of a transient HS domain, which relaxed back to the initial phase within a few seconds. Actually, at this area of the crystal we could observe a propagating domain only for rather high laser pulse energies - close to the laser damage threshold (see the Supplemental Material [23]). The observation of such transient HS domains within the hysteresis region is rather surprising, especially if one takes into account their large volume $\left(\sim 10 \times 12 \times 6 \mu \mathrm{m}^{3}\right)$ and long lifetime ( $c a .30 \mathrm{sec})$.

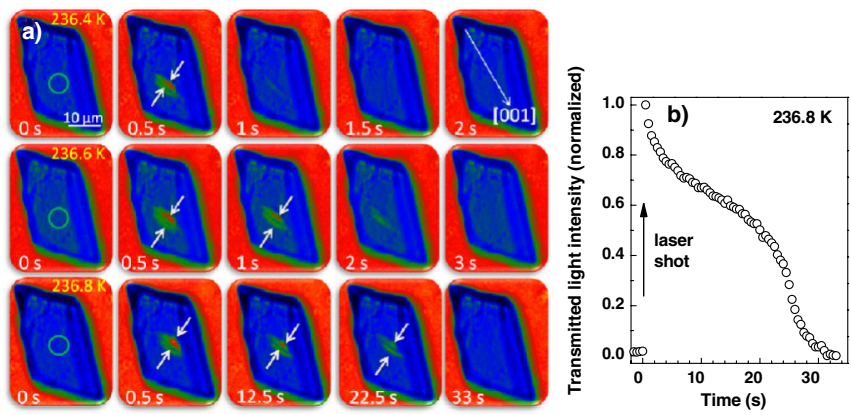

FIG. 3 (color online). Relaxation of the laser-induced high spin domain. (a) Bright-field, transmission optical microscopy snapshots of a crystal of $\mathbf{1}$ before and after a single laser shot (4 ns, $532 \mathrm{~nm}, 0.1 \mu \mathrm{J}, 3 \mu \mathrm{m}$ spot size) at three different temperatures in the heating branch. The arrows depict the relaxation direction, and the circles indicate the area of the laser spots. Dark gray (blue) and light gray (red) (artificial) colors refer to the IP and the HS phase, respectively. (The movies are available in the Supplemental Material [23].) (b) Relaxation curve of the metastable HS domain at $236.8 \mathrm{~K}$.
Figure 3 shows also experiments where the crystal was irradiated by the same laser energy at (nearly) the same place but at different temperatures. One can see the characteristic slow-down of the relaxation when approaching the phase transition [25,26]. Another important observation is that in each case a similar, ellipsoidal HS domain is formed with a major (minor) axis of $c a$. 12(6) $\mu \mathrm{m}$, respectively-in contrast to the nearly Gaussian shape of the diffractionlimited laser spot [also shown in Fig. 3(a)]. The formation of such elongated nuclei is predicted by classical nucleation theory, as this shape allows for the minimization of the interface and elastic strain energy [27]. In this respect, it is important to notice also that the orientation of the laserinduced domains is not arbitrary but instead very well reproducible, the major axis being parallel with the monoclinic $c$ axis of the crystal. Interestingly, during the relaxation the aspect ratio of the HS domains tends to further increase. The relaxation kinetics is depicted in Fig. 3(b) in which the well-known sigmoidal relaxation curve is observed. Such self-accelerating behavior is typical for many cooperative SCO systems and results from the strong electron-lattice coupling [5].

In certain cases, it was also possible to induce a HS domain, which remained stable for (at least) several hours (see the Supplemental Material for a few examples [23]). In general, we found that if a domain does not relax or continue to grow for at least a few minutes, it will be stable "infinitely" and an increase of the sample temperature is needed to complete the transition. The formation of such stable HS domains may arise from the trapping of the phase boundary by defect sites, which can form an obstacle for the further propagation or relaxation of the domain. We shall also mention that we have not been able to find unambiguous evidence for the laser-induced reverse switch $(\mathrm{HS} \rightarrow \mathrm{IP}$ ) in this material on the descending branch of the thermal hysteresis loop. To achieve this reverse switch, it would be very important to extend this investigation to shorter time scales in order to better assess the mechanism of the first stages of the laser-induced transition.

These remarkable and largely unpredicted spatiotemporal scenarios can be understood from simple thermodynamical considerations. Figure 4 depicts the free energy of the crystal within the irradiated volume before and after a laser shot, calculated using the well-known Ising-like model in the mean-field approximation $[28,29]$. We took into account the effect of local elastic stress on the nucleated droplet as a modification of the energy gap between the HS and LS states (for calculation details, see the Supplemental Material [23]). Before the application of the laser pulse, the free energy of the molecules inside the crystal has a double well form corresponding to the bistability phenomenon within the hysteresis loop. When the system is close to the ascending branch of the hysteresis, the IP is metastable whereas the HS phase is thermodynamically stable. The application of the laser pulse leads to the switching of the molecules from the 


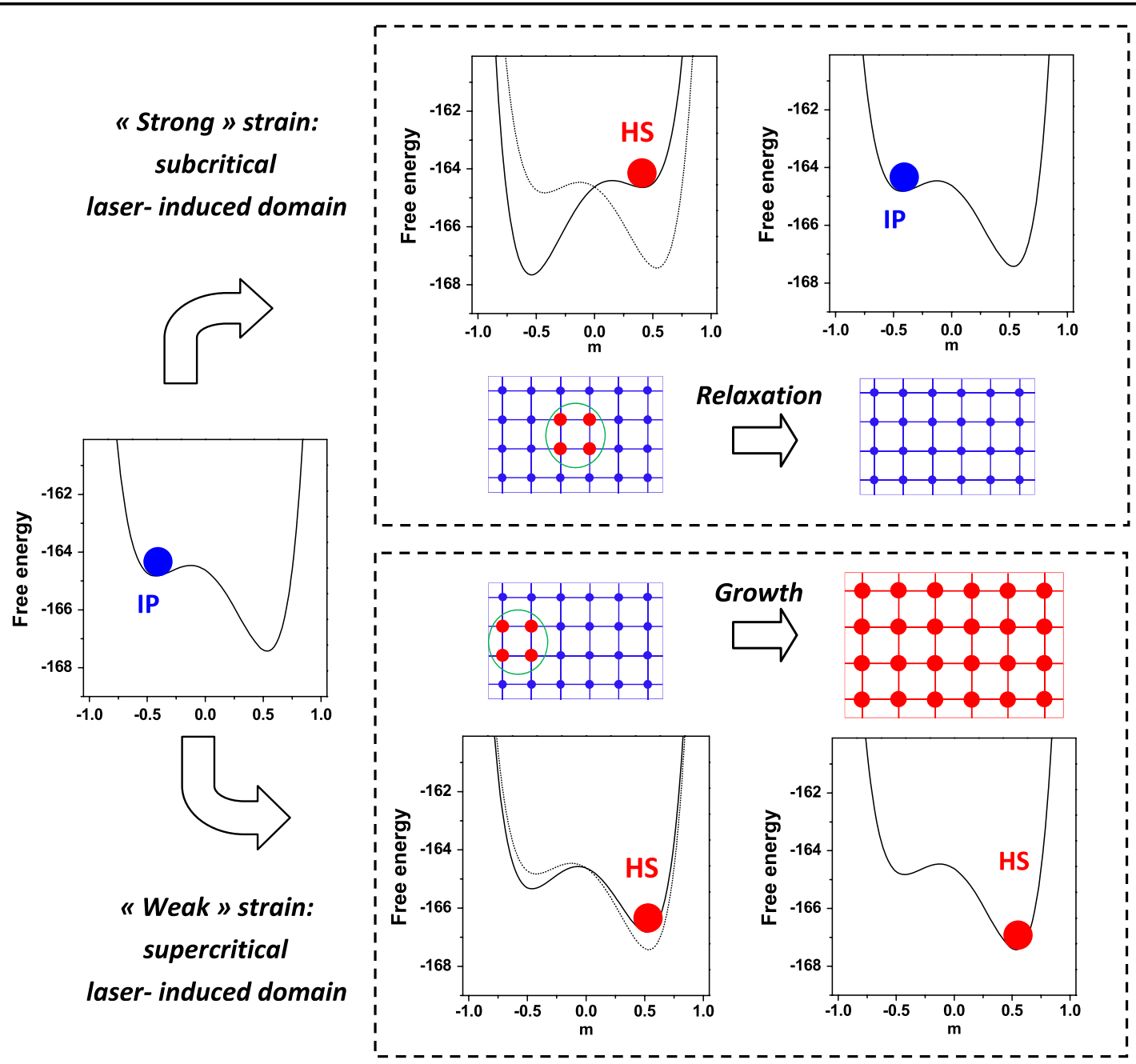

FIG. 4 (color online). Simulation of the free energy of the crystal within the irradiated volume before and after a laser shot using the Ising-like model in the mean-field approximation. The system is initially in the metastable IP (2/3 LS molecules). The focused laser pulse brings the system to the HS state within the irradiated volume but it changes also the free energy of the two phases due to the accommodation strain. Depending on the magnitude of the elastic strain, the laser-induced HS domain can be therefore either stable (bottom panel) or metastable (top panel).

LS to the HS state through various mechanisms (optical, elastic, and thermal switching), which are not resolved in our experiment. After this highly nonequilibrium process, a finite size HS nucleus is formed in or around the photoexcited volume. This nucleus is embedded in the parent phase (IP) and it is therefore destabilized with respect to an entirely HS crystal in the same thermodynamical conditions due to the interface and elastic strain energies. These energies scale with $r^{2}$ and $r^{3}$, respectively; therefore, at large sizes (which is our case) the latter will dominate [27]. This lattice strain arises chiefly due to the significant volume mismatch between the IP and the HS phase $(\Delta V / V=2.5 \%)$ [17]. During the phase transition, the lattice has to accommodate therefore a considerable strain, which will alter the energy of the phases in a rather inhomogenous manner. Because of this accommodation strain, the laser-induced HS domain will always be destabilized to some extent. If the destabilization is relatively small, the HS phase remains the stable phase. In the language of classical nucleation theory, it means that the volume of the nucleus exceeds the critical size, and therefore the daughter phase will grow and the parent phase shrinks. On the other hand, if the initial HS nucleus is more strongly destabilized by the elastic strain it becomes metastable (i.e., subcritical) and it will be therefore redissolved in the parent phase after the dissipation of the laser-induced heat.

Whether the laser-induced HS nucleus redissolves or continues to grow depends primarily on the sample temperature and on the laser pulse characteristics. However, even in identical experimental conditions, we observed different behaviors depending on which part of the crystal was irradiated by the laser pulse. Obviously, this observation is related to the finite size as well as to the 
inhomogenity of the crystal. In particular, the accommodation strain will be different in the middle of the crystal or close to its edges where the interface with the parent phase is reduced (for the same nucleated volume). Furthermore, in real crystals the strain varies also because the elastic properties are locally different and the distribution of crystal defects is inhomogenous. For these reasons, the energy barriers between the two phases can be locally very different. The effect of the laser pulse becomes thus spatially dependent and more generally sample dependent.

In summary, the effect of a focused laser pulse on a comparatively large single crystal of a molecular spincrossover compound has been investigated close to the thermal spin transition using time-resolved optical microscopy imaging. These experiments revealed very important new facets of the spatiotemporal dynamics of the photoinduced phase transition. We have shown that a spatially localized laser pulse can trigger the transition of the whole crystal via a stress-driven cooperative process. Remarkably, in the same experimental conditions we observed either a subcritical or a supercritical laser-induced domain depending on the irradiated region within the crystal, which was attributed primarily to the different accommodation strain. This observation of subcritical transient domains provides not only new insights of the role of local elastic strain but also opens unpredicted perspectives for ultrafast pump-probe experiments within the hysteresis loop. Indeed, the spatiotemporal evolution of the system was found well reproducible in each case-providing thus an appealing scope for material and process engineering as well as for the development of applications where the state of the system is controlled by a weak photoexcitation. These observations have broad implications for the field of PIPT. They reveal that the stress-driven phase boundary motion can be a very efficient but at the same time a rather sluggish stage of the overall switching process in cooperative systems. The results suggest also very interesting size-dependent phenomena in these materials depending on the relative size of the laser-induced domain and that of the crystal.

This work was supported by Project No. ANR-10-BLAN716-1. S. Bedoui thanks the Ministry of Higher Education and Scientific Research of Tunisia for a $\mathrm{PhD}$ grant.

*gabor.molnar@1cc-toulouse.fr

azzedine.bousseksou@lcc-toulouse.fr

[1] S. Koshihara, Y. Tokura, T. Mitani, G. Saito, and T. Koda, Phys. Rev. B 42, 6853 (1990).

[2] E. Collet et al., Science 300, 612 (2003).

[3] H. Ichikawa et al., Nature Mater. 10, 101 (2011).

[4] Photoinduced Phase Transitions, edited by K. Nasu (World Scientific, Singapore, 2004).
[5] P. Gütlich, A. Hauser, and H. Spiering, Angew. Chem., Int. Ed. Engl. 33, 2024 (1994).

[6] S. Decurtins, P. Gütlich, C.P. Köhler, and H. Spiering, Chem. Phys. Lett. 105, 1 (1984).

[7] E. Freysz, S. Montant, S. Létard, and J.-F. Létard, Chem. Phys. Lett. 394, 318 (2004).

[8] S. Bonhommeau, G. Molnár, A. Galet, A. Zwick, J.-A. Real, J. J. McGarvey, and A. Bousseksou, Angew. Chem., Int. Ed. Engl. 44, 4069 (2005).

[9] S. Cobo, D. Ostrovskii, S. Bonhommeau, L. Vendier, G. Molnár, L. Salmon, K. Tanaka, and A. Bousseksou, J. Am. Chem. Soc. 130, 9019 (2008).

[10] N. O. Moussa, D. Ostrovskii, V. Martinez Garcia, G. Molnár, K. Tanaka, A. B. Gaspar, J.A. Real, and A. Bousseksou, Chem. Phys. Lett. 477, 156 (2009).

[11] M. Lorenc et al., Phys. Rev. Lett. 103, 028301 (2009).

[12] M. Lorenc et al., Phys. Rev. B 85, 054302 (2012).

[13] G. Gallé, D. Deldicque, J. Degert, Th. Forestier, J.-F. Létard, and E. Freysz, Appl. Phys. Lett. 96, 041907 (2010).

[14] G. Gallé, J. Degert, C. Mauriac, C. Etrillard, J. F. Letard, and E. Freysz, Chem. Phys. Lett. 500, 18 (2010).

[15] O. Fouché, J. Degert, G. Jonusauskas, N. Daro, J.-F. Létard, and E. Freysz, Phys. Chem. Chem. Phys. 12, 3044 (2010).

[16] H. Liu, A. Fujishima, and O. Sato, Appl. Phys. Lett. 86, 122511 (2005).

[17] S. Bonnet, M. A. Siegler, J. Sánchez Costa, G. Molnár, A. Bousseksou, A. L. Spek, P. Gamez, and J. Reedijk, Chem. Commun. (Cambridge) 43 (2008) 5619.

[18] S. Bonnet, G. Molnár, J. Sanchez Costa, M. A. Siegler, A. L. Spek, A. Bousseksou, W.-T. Fu, P. Gamez, and J. Reedijk, Chem. Mater. 21, 1123 (2009).

[19] H. J. Shepherd, S. Bonnet, P. Guionneau, S. Bedoui, G. Garbarino, W. Nicolazzi, A. Bousseksou, and G. Molnár, Phys. Rev. B 84, 144107 (2011).

[20] S. Bedoui, G. Molnár, S. Bonnet, C. Quintero, H. J. Shepherd, W. Nicolazzi, L. Salmon, and A. Bousseksou, Chem. Phys. Lett. 499, 94 (2010).

[21] S. Bedoui, M. Lopes, S. Zheng, S. Bonnet, G. Molnár, and A. Bousseksou, Adv. Mater. 24, 2475 (2012).

[22] A. Slimani, F. Varret, K. Boukheddaden, C. Chong, H. Mishra, J. Haasnoot, and S. Pillet, Phys. Rev. B 84, 094442 (2011).

[23] See Supplemental Material at http://link.aps.org/ supplemental/10.1103/PhysRevLett.109.135702 for details of the theoretical model as well as for additional experimental results.

[24] S. C. Ngan and L. Truskinovsky, J. Mech. Phys. Solids 47, 141 (1998).

[25] H. Watanabe, H. Hirori, G. Molnár, A. Bousseksou, and K. Tanaka, Phys. Rev. B 79, 180405(R) (2009).

[26] I. Krivokapic, C. Enachescu, R. Bronisz, and A. Hauser, Inorg. Chim. Acta 361, 3616 (2008).

[27] B. Revzin, J. Pelleg, and G. Gorodetsky, Phys. Rev. B 59, 9144 (1999).

[28] A. Bousseksou, J. Nasser, J. Linares, K. Boukheddaden, and F. Varret, J. Phys. I 2, 1381 (1992).

[29] S. Bonhommeau, G. Molnár, M. Goiran, K. Boukheddaden, and A. Bousseksou, Phys. Rev. B 74, 064424 (2006). 